\title{
CONDITIONS AUX LIMITES APPROCHÉES POUR LES COUCHES MINCES PÉRIODIQUES
}

\author{
Habib Ammari ${ }^{1}$ et Chiraz Latiri-Grouz ${ }^{1}$
}

\begin{abstract}
Approximate boundary conditions for the scattering problem by a penetrable obstacle coated with a thin periodic layer are derived.

Résumé. Nous écrivons et nous justifions des conditions aux limites approchées pour des couches minces périodiques recouvrant un objet parfaitement conducteur en polarisation transverse électrique et transverse magnétique.
\end{abstract}

AMS Subject Classification. 78A45, 35B27, 35C20.

Reçu : 8 Octobre 1997. Révisé : 8 Juillet 1998.

\section{INTRODUCTION}

Les conditions aux limites approchées permettent de représenter un objet recouvert d'une couche mince de matériau diélectrique ou magnétique. La méthode consiste à remplacer la couche mince par une condition aux limites approchées qui donne une bonne approximation du champ diffracté par l'objet à l'extérieur de la couche. La procédure permet d'accélérer ou de rendre possible les calculs numériques du champ à l'extérieur de la couche mince. Cette idée a été introduite en électromagnétisme dans les années quarante par Shukin-Léontovitch pour traiter de la diffraction par des objets diélectriques à fort indice et à pertes. Elle a été ensuite appliquée pour approcher le problème de diffraction par des couches minces par de très nombreux auteurs parmi lesquels nous citons Harrington-Mautz [17], Karp-Karal [18], King-Wait [19], Senior-Volakis [21] et plus récemment, EngquistNédélec [16], Bendali-Vernhet [13], Bendali-Lemrabet [14] et l'un des auteurs de cet article [1-11]. Dans cet article nous écrivons des conditions aux limites approchées pour les couches minces périodiques à l'aide d'un développement asymptotique à deux échelles et nous démontrons des estimations d'erreur. A notre connaissance ces conditions aux limites approchées sont nouvelles. Notre approche est différente de celle adoptée dans [1] pour l'écriture de conditions aux limites approchant l'effet d'un réseau d'inclusions parfaitement conductrices dans une couche mince de coefficients constants sur la diffraction d'une onde électromagnétique. L'approche adoptée dans [1] consiste à écrire les conditions d'impédance généralisées à partir des correcteurs de couches limites exhibés pour corriger les conditions de transmission à travers l'interface diélectrique-milieu extérieur et les conditions limites sur le bord des inclusions parfaitement conductrices.

\footnotetext{
Keywords and phrases. Approximate boundary conditions, Helmholtz equation, thin periodic coatings, homogenization.

${ }^{1}$ Centre de Mathématiques Appliquées, CNRS UMR 7641, École Polytechnique, 91128 Palaiseau Cedex, France.

e-mail: ammari@cmapx.polytechnique.fr, latiri@cmapx.polytechnique.fr
} 
Dans cet article, nous traitons une famille d'équations de la forme :

$$
\operatorname{div}\left(\frac{1}{\mu} \nabla u\right)+k^{2} \varepsilon u=0
$$

où $\mu \mathrm{ou} /$ et $\varepsilon$ seraient périodiques dans la couche mince (suivant la variable tangentielle avec un période de même ordre que l'épaisseur de la couche).

Le plan de cet article est le suivant :

Dans la première partie, nous écrivons des conditions aux limites approchées pour une couche mince dont la perméabilité magnétique est variable. La méthode consiste à se ramener par un changement d'échelles (scaling) à un problème posé dans un domaine fixe. Ces conditions aux limites approchées généralisent celles écrites et rigoureusement justifiées dans $[14,16]$ pour une couche mince à coefficients constants.

La deuxième partie est consacrée aux couches minces périodiques. Nous commençons par écrire formellement une classe de conditions aux limites approchées. Nous donnons ensuite des estimations d'erreur optimales. Ce qui nous permet de justifier l'écriture formelle de ces conditions approchées ainsi que celles trouvées dans la section précédente. L'idée, dans le cas périodique, est d'homogénéiser la couche après changement d'échelles. Les difficultés rencontrées dans notre analyse sont d'une part la stabilité de la solution par rapport à l'épaisseur de la couche, due au fait que le problème est un problème de perturbation singulière, et d'autre part les variations rapides de la perméabilité magnétique (ou de la permittivité électrique) de la couche mince dans la direction tangentielle.

Dans la troisième partie, nous traitons le cas où le coefficient $\mu$ est rapidement variable. Les conditions aux limites approchées sont obtenues dans ce cas à partir d'une analyse asymptotique d'un certain opérateur pseudo-différentiel décrivant la diffraction dans la couche mince de diélectrique.

\section{COUCHES MINCES}

\subsection{Cas d'une condition aux limites de type Neumann}

Soit $\delta$ un paramètre destiné à tendre vers zéro, et qui décrit dans un premier temps l'épaisseur de la couche mince. Soit $\Omega$ un ouvert borné régulier de $\mathbb{R}^{2}$ qui représente un objet diffractant, $\Gamma=\partial \Omega$ son bord supposé de classe $\mathcal{C}^{\infty}$. Soit $\Omega_{i}^{\delta}=\{x \in \Omega$ tel que $d(x, \Gamma)<\delta\}$ la couche mince, où $d$ est la distance du point $x$ au bord $\Gamma$. En utilisant les notations classiques en géométrie différentielle [20], on introduit une paramétrisation locale de $\partial \Omega$, classiquement notée $(s, n)$. On a alors $\left.\Omega_{i}^{\delta}=\partial \Omega \times\right]-\delta, 0\left[\right.$. Enfin, nous notons $\Omega_{e}$ le complémentaire de $\bar{\Omega}$ dans $\mathbb{R}^{2}$.

Nous considérons le problème de transmission représentant la diffraction d'une onde électromagnétique par la couche mince $\Omega_{i}^{\delta}$ en polarisation TE (transverse électrique) :

$$
\left\{\begin{array}{l}
\Delta u_{i}^{\delta}+k^{2} q\left(s, \frac{n}{\delta}\right) u_{i}^{\delta}=0 \quad \text { dans } \Omega_{i}^{\delta} \\
\Delta u_{e}^{\delta}+k^{2} u_{e}^{\delta}=0 \quad \text { dans } \Omega_{e} \\
\partial_{n} u_{i}^{\delta}=0 \quad \operatorname{sur}\{n=-\delta\} \\
\left.u_{i}^{\delta}\right|_{n \rightarrow 0^{-}}=\left.u_{e}^{\delta}\right|_{n \rightarrow 0^{+}},\left.\partial_{n} u_{i}^{\delta}\right|_{n \rightarrow 0^{-}}=\left.\partial_{n} u_{e}^{\delta}\right|_{n \rightarrow 0^{+}}, \\
u_{e}^{\delta}-u^{\text {in }} \text { satisfait la condition de radiation sortante }
\end{array}\right.
$$

où $u^{\text {in }}$ est une onde incidente plane, $k$ est une constante strictement positive et $q(s, n / \delta)$ est une fonction de la variable $s$ et $n / \delta$. Ce problème correspond à la diffraction d'une onde électromagnétique par la couche mince $\Omega_{i}^{\delta}$ en polarisation TE. Notre objectif est de construire un développement asymptotique de $u_{e}^{\delta}$ lorsque $\delta$ tend vers zéro. 
Pour cela, on effectue un Ansatz dans la couche mince $\Omega_{i}^{\delta}$ du type :

$$
u_{i}^{\delta}(s, n)=u_{i}^{0}(s, y)+\delta u_{i}^{1}(s, y)+\delta^{2} u_{i}^{2}(s, y)+\ldots,
$$

où la variable supplémentaire $y=n / \delta$. A l'extérieur de la couche $\Omega_{i}^{\delta}$, on pose (dans un voisinage de $\partial \Omega$ si $\Omega$ n'est pas convexe) :

$$
u_{e}^{\delta}(s, n)=u_{e}^{0}(s, n)+\delta u_{e}^{1}(s, n)+\delta^{2} u_{e}^{2}(s, n)+\ldots
$$

L'opérateur Laplacien s'écrit en coordonnées locales :

$$
\Delta=\frac{1}{1+c(s) n} \partial_{s}\left(\frac{1}{1+c(s) n} \partial_{s}\right)+\partial_{n}^{2}+c(s, n) \partial_{n} .
$$

Appliqué à $u_{i}^{j}$ l'opérateur Laplacien devient

$$
\frac{1}{\delta^{2}} \partial_{y^{2}}^{2}+\frac{1}{\delta} c(s, \delta y) \partial_{y}+\frac{1}{1+\delta c(s) y} \partial_{s}\left(\frac{1}{1+\delta c(s) y} \partial_{s}\right),
$$

où $c(s, n)$ est la courbure au point $(s, n)$ et $c(s)=c(s, 0)$. Ceci permet après identification des coefficients de même puissance de $\delta$ d'obtenir :

$$
\left\{\begin{array}{l}
\partial_{y}^{2} u_{i}^{0}=0 \\
\partial_{y}^{2} u_{i}^{1}+c(s) \partial_{y} u_{i}^{0}=0 \\
\partial_{y}^{2} u_{i}^{2}+c(s) \partial_{y} u_{i}^{1}+\partial_{s}^{2} u_{i}^{0}+\partial_{n} c(s, 0) y \partial_{y} u_{i}^{0}+k^{2} q(s, y) u_{i}^{0}=0 \\
\vdots
\end{array}\right.
$$

Pour construire des conditions aux limites approchées, notre méthode consiste à chercher des relations approchées entre d'une part $\left.\partial_{n} u_{e}^{\delta}\right|_{n \rightarrow 0^{+}}$et d'autre part $\left.u_{e}^{\delta}\right|_{n \rightarrow 0^{+}}$et ses dérivées par rapport à la variable tangentielle $s$. On a d'après les conditions de transmission dans (2) :

$$
\left\{\begin{array}{l}
\left.u_{e}^{\delta}\right|_{n \rightarrow 0^{+}}=\left.u_{i}^{0}(s, y)\right|_{y=0}+\left.\delta u_{i}^{1}(s, y)\right|_{y=0}+\left.\delta^{2} u_{i}^{2}(s, y)\right|_{y=0}+\ldots \\
\left.\partial_{n} u_{e}^{\delta}\right|_{n \rightarrow 0^{+}}=\left.\frac{1}{\delta} \partial_{y} u_{i}^{0}(s, y)\right|_{y=0}+\left.\partial_{y} u_{i}^{1}(s, y)\right|_{y=0}+\ldots
\end{array}\right.
$$

Les équations (5) avec les conditions aux limites $\left.\partial_{y} u_{i}^{j}(s, y)\right|_{y=-1}=0$ conduisent à

$$
\left\{\begin{aligned}
u_{i}^{0}(s, y)= & \left.u_{i}^{0}(s, y)\right|_{y=0} \\
u_{i}^{1}(s, y)= & \left.u_{i}^{1}(s, y)\right|_{y=0} \\
u_{i}^{2}(s, y)= & -\left.\frac{1}{2} y(y+2) \partial_{s}^{2} u_{i}^{0}\right|_{y=0}-\left.k^{2}\left(\int_{-1}^{y} \int_{-1}^{y^{\prime}} q\left(s, y^{\prime \prime}\right) \mathrm{d} y^{\prime \prime} \mathrm{d} y^{\prime}\right) u_{i}^{0}\right|_{y=0} \\
& +\left.u_{i}^{2}\right|_{y=0}+\left.k^{2}\left(\int_{-1}^{0} \int_{-1}^{y^{\prime}} q\left(s, y^{\prime \prime}\right) \mathrm{d} y^{\prime \prime} \mathrm{d} y^{\prime}\right) u_{i}^{0}\right|_{y=0}
\end{aligned}\right.
$$


Si on définit $u_{i}^{\delta, j}$ (resp. $u_{e}^{\delta, j}$ ) comme le développement asymptotique tronqué à l'ordre $j$ de celui de $u_{i}^{\delta}$ (resp. $u_{e}^{\delta}$ ) donné par (3) (resp. (4)):

$$
\begin{aligned}
& u_{i}^{\delta, j}(s, n)=\left.u_{i}^{0}(s, y)\right|_{y=n / \delta}+\left.\delta u_{i}^{1}(s, y)\right|_{y=n / \delta}+\ldots+\left.\delta^{j} u_{i}^{j}(s, y)\right|_{y=n / \delta} \\
& u_{e}^{\delta, j}(s, n)=u_{e}^{0}(s, n)+\delta u_{e}^{1}(s, n)+\ldots+\delta^{j} u_{e}^{j}(s, n)
\end{aligned}
$$

les identités $(6,7)$ donnent

$$
\partial_{n} u_{i}^{\delta, 1}=\partial_{n}\left(u_{i}^{0}+\delta u_{i}^{1}\right)=\frac{1}{\delta} \partial_{y}\left(u_{i}^{0}+\delta u_{i}^{1}\right)=0(\delta) \quad \operatorname{sur} \partial \Omega
$$

et de même, on obtient

$$
\partial_{n} u_{i}^{\delta, 2}+\delta k^{2} \tilde{q}(s) u_{i}^{\delta, 2}+\delta \partial_{s}^{2} u_{i}^{\delta, 2}=0\left(\delta^{2}\right) \quad \operatorname{sur} \partial \Omega,
$$

où le coefficient de perméabilité magnétique effectif est donné par

$$
\tilde{q}(s)=\int_{-1}^{0} q(s, y) \mathrm{d} y .
$$

La relation précédente conduit à

$$
\partial_{n} u_{e}^{\delta, 2}+\delta k^{2} \tilde{q}(s) u_{e}^{\delta, 2}+\delta \partial_{s}^{2} u_{e}^{\delta, 2}=0\left(\delta^{2}\right) \quad \text { sur } \partial \Omega .
$$

Maintenant, pour obtenir des conditions aux limites approchées pour $\Omega_{i}^{\delta}$, nous remplaçons le problème de transmission (2) par l'équation de Helmholtz suivante à l'extérieur de la couche :

$$
\left\{\begin{array}{l}
\Delta \tilde{u}_{e}^{\delta, j}+k^{2} \tilde{u}_{e}^{\delta, j}=0 \quad \text { dans } \Omega_{e} \\
\tilde{u}_{e}^{\delta, j}-u^{\text {in }} \text { satisfait la condition de radiation sortante }
\end{array}\right.
$$

avec les conditions aux limites :

- Condition aux limites approchée d'ordre 0 :

$$
\partial_{n} \tilde{u}_{e}^{\delta, 1}=0 \quad \text { sur } \partial \Omega .
$$

- Condition aux limites approchée d'ordre 1 :

\section{Remarque 2.1.}

$$
\partial_{n} \tilde{u}_{e}^{\delta, 2}+\delta k^{2} \tilde{q}(s) \tilde{u}_{e}^{\delta, 2}+\delta \partial_{s}^{2} \tilde{u}_{e}^{\delta, 2}=0 \quad \text { sur } \partial \Omega .
$$

- Notons que la fonction $\tilde{u}_{e}^{\delta, j}$ n'est pas exactement $u_{e}^{\delta, j}$. Ce que nous proposons dans toute notre étude, c'est plutôt le calcul du second développement que le premier. La raison est qu'il paraît plus facile de traiter numériquement la seconde approche en résolvant les différents problèmes vérifiées par $u_{0}, u_{1}, \ldots$ que de traiter l'unique problème (11) qui présente quelques instabilités numériques dues au fait que ce soit un problème de perturbation singulière [12].

- Lorsque la fonction $q$ est constante dans $\Omega_{i}^{\delta}$, l'identité $(10)$ donne $\tilde{q}(s)=q$. Nous retrouvons alors les conditions aux limites approchées d'ordre un écrites dans $[14,16]$. 


\subsection{Cas d'une condition aux limites de type Dirichlet}

Le traitement d'une condition aux limites de type Dirichlet sur $\{n=-\delta\}$ qui correspond à une polarisation transverse magnétique de l'onde incidente est légèrement différent. Les développements asymptotiques supposés (3) et $(4)$ de $\left(u_{e}^{\delta}(s, n), u_{i}^{\delta}(s, n)\right)$ restent valables. En utilisant les équations (5) avec les conditions aux limites de type Dirichlet, on obtient :

$$
\left\{\begin{array}{l}
u_{i}^{0}(s, y)=\left.(y+1) \partial_{y} u_{i}^{0}(s, y)\right|_{y=0} \\
u_{i}^{1}(s, y)=\left.(y+1) u_{i}^{1}(s, y)\right|_{y=0}+\left.\frac{c(s)}{2}\left(y^{2}+1\right) \partial_{y} u_{i}^{0}(s, y)\right|_{y=0}, \\
\partial_{y}^{2} u_{i}^{2}(s, y)=-\left.(y+1) \partial_{s}^{2} \partial_{y} u_{i}^{0}\right|_{y=0}-\left.c(s) \partial_{y} u_{i}^{1}(s, y)\right|_{y=0} \\
\quad-\left.c(s) \partial_{y} u_{i}^{0}(s, y)\right|_{y=0}-\partial_{n} c(s, 0) y \partial_{y} u_{i}^{0}-\left.k^{2} q(s, y)(y+1) \partial_{y} u_{i}^{0}\right|_{y=0} .
\end{array}\right.
$$

Un calcul analogue au cas d'une condition aux limites de type Neumann permet de trouver la condition aux limites approchée d'ordre deux qui s'écrit sur $\partial \Omega$ :

$$
\left(1+\frac{\delta}{2} c(s)\right) \tilde{u}_{e}^{\delta, 2}+\delta \partial_{n} \tilde{u}_{e}^{\delta, 2}+\frac{\delta^{2}}{3}\left\{-\partial_{s^{2}}^{2} \tilde{u}_{e}^{\delta, 2}-\left(\frac{1}{4} c^{2}(s)-\partial_{n} c(s, 0)-k^{2} \tilde{q}(s)\right) \tilde{u}_{e}^{\delta, 2}\right\}=0,
$$

et de montrer que la perméabilité magnétique effective qui intervient dans cette condition approchée n'est plus la moyenne de la fonction $q(s, y)$ par rapport à la variable $y$ mais elle est donnée par l'expression suivante (voir $[6])$ :

$$
\tilde{q}(s)=-3 \int_{-1}^{0}\left(\int_{-1}^{y} q\left(s, y^{\prime}\right)\left(y^{\prime}+1\right) \mathrm{d} y^{\prime}\right) \mathrm{d} y+3 \int_{-1}^{0} q(s, y)(y+1) \mathrm{d} y .
$$

\section{COUChes Minces PÉRIOdiques}

Dans cette section, nous allons écrire formellement des conditions aux limites approchées pour une couche mince de perméabilité magnétique périodique en la variable tangentielle $s$ de période de l'ordre de l'épaisseur de la couche. Nous gardons les mêmes notations que dans la section précédente à savoir : $\Omega$ un ouvert borné régulier de $\mathbb{R}^{2},(s, n)$ une paramétrisation locale de son bord $\partial \Omega$ supposé de classe $\left.\mathcal{C}^{\infty}, \Omega_{i}^{\delta}=\partial \Omega \times\right]-\delta, 0\left[\right.$ et $\Omega_{e}$ le complémentaire de $\bar{\Omega}$ dans $\mathbb{R}^{2}$.

\subsection{Cas d'une condition aux limites de type Neumann}

Nous considérons le problème de transmission :

$$
\left\{\begin{array}{l}
\Delta u_{i}^{\delta}+k^{2} q\left(s, \frac{s}{\delta}, \frac{n}{\delta}\right) u_{i}^{\delta}=0 \quad \text { dans } \Omega_{i}^{\delta} \\
\Delta u_{e}^{\delta}+k^{2} u_{e}^{\delta}=0 \quad \text { dans } \Omega_{e} \\
\partial_{n} u_{i}^{\delta}=0 \quad \operatorname{sur}\{n=-\delta\} \\
\left.u_{i}^{\delta}\right|_{n \rightarrow 0^{-}}=\left.u_{e}^{\delta}\right|_{n \rightarrow 0^{+}},\left.\partial_{n} u_{i}^{\delta}\right|_{n \rightarrow 0^{-}}=\left.\partial_{n} u_{e}^{\delta}\right|_{n \rightarrow 0^{+}} \\
u_{e}^{\delta}-u^{\text {in }} \text { satisfait la condition de radiation sortante }
\end{array}\right.
$$

où le coefficient de perméabilité magnétique $q$ est périodique en la variable $s / \delta$ de période un et égal à 1 à l'extérieur de la couche $\Omega_{i}^{\delta}$. Nous allons écrire des conditions aux limites approchées pour la couche mince périodique $\Omega_{i}^{\delta}$. Nous effectuons l'Ansatz

$$
u_{i}^{\delta}(s, n)=u_{i}^{0}(s, x, y)+\delta u_{i}^{1}(s, x, y)+\delta^{2} u_{i}^{2}(s, x, y)+\ldots,
$$


où $x=s / \delta, y=n / \delta$ et $u_{i}^{j}$ est périodique en la variable supplémentaire $x=s / \delta$ de période 1 pour tout entier naturel $j$ et nous posons

$$
u_{e}^{\delta}(s, n)=u_{e}^{0}(s, n)+\delta u_{e}^{1}(s, n)+\delta^{2} u_{e}^{2}(s, n)+\ldots,
$$

à l'extérieur de la couche mince périodique $\Omega_{i}^{\delta}$. Nous introduisons

$$
v_{i}^{\delta}(s, n)=u_{i}^{\delta}(s, n)-\left.u_{e}^{\delta}(s, n)\right|_{n=0},
$$

et nous effectuons de même l'Ansatz

$$
v_{i}^{\delta}(s, n)=v_{i}^{0}(s, x, y)+\delta v_{i}^{1}(s, x, y)+\delta^{2} v_{i}^{2}(s, x, y)+\ldots,
$$

où $y=n / \delta$. Appliquée à $v_{i}^{j}$ l'opérateur $\partial_{s}$ devient $\partial_{s}+\frac{1}{\delta} \partial_{x}$ et l'opérateur $\partial_{n}$ devient $\frac{1}{\delta} \partial_{y}$. En utilisant l'Ansatz :

$$
\left.u_{e}^{\delta}(s, n)\right|_{n=0}=\left.u_{e}^{0}(s, n)\right|_{n=0}+\left.\delta u_{e}^{1}(s, n)\right|_{n=0}+\ldots
$$

et l'expression du Laplacien en coordonnées locales $(s, n)$

$$
\Delta=\frac{1}{1+c(s) n} \partial_{s}\left(\frac{1}{1+c(s) n} \partial_{s}\right)+\partial_{n}^{2}+c(s, n) \partial_{n}
$$

qui devient

$$
\begin{aligned}
\frac{1}{\delta^{2}}\left[\frac{1}{(1+\delta c(s) y)^{2}} \partial_{x}^{2}+\partial_{y}^{2}\right]+\frac{1}{\delta}[ & \left.\frac{1}{(1+\delta c(s) y)^{2}} \partial_{x} \partial_{s}+\frac{1}{1+\delta c(s) y} \partial_{s}\left(\frac{1}{1+\delta c(s) y} \partial_{x}\right)+c(s, \delta y) \partial_{y}\right] \\
& +\frac{1}{1+\delta c(s) y} \partial_{s}\left(\frac{1}{1+\delta c(s) y} \partial_{s}\right)
\end{aligned}
$$

nous obtenons après identification des termes en $\delta^{-2}$ l'équation

$$
\left(\partial_{x}^{2}+\partial_{y}^{2}\right) v_{i}^{0}=0
$$

Ce qui donne en utilisant la périodicité de $v_{i}^{0}$ en $x$ et les conditions aux limites en $y=0$ et $y=-1$ que $v_{i}^{0}$ est nulle. Le terme $v_{i}^{1}$ vérifie de même l'équation :

$$
\left(\partial_{x}^{2}+\partial_{y}^{2}\right) v_{i}^{1}=0
$$

L'identification des termes en $\delta^{0}$ donne compte-tenu du fait que $v_{i}^{0}=v_{i}^{1}=0$ l'équation :

$$
\left(\partial_{x}^{2}+\partial_{y}^{2}\right) v_{i}^{2}=-\left.\partial_{s}^{2} u_{e}^{0}(s, n)\right|_{n=0}-\left.k^{2} q(s, x, y) u_{e}^{0}(s, n)\right|_{n=0},
$$

posée dans le carré $] 0,1[\times]-1,0\left[\right.$. Soient $\tilde{u}_{i}^{j}(s, y)$ la moyenne par rapport à la variable supplémentaire $x$ de la fonction $u_{i}^{j}(s, x, y)$ :

$$
\tilde{u}_{i}^{j}(s, y)=\int_{0}^{1} u_{i}^{j}(s, x, y) \mathrm{d} x
$$


$\tilde{u}_{i}^{\delta, j}$ défini par

$$
\tilde{u}_{i}^{\delta, j}(s, y)=\tilde{u}_{i}^{0}(s, y)+\delta \tilde{u}_{i}^{1}(s, y)+\ldots+\delta^{j} \tilde{u}_{i}^{j}(s, y)
$$

et

$$
u_{e}^{\delta, j}(s, n)=u_{e}^{0}(s, n)+\delta u_{e}^{1}(s, n)+\ldots+\delta^{j} u_{e}^{j}(s, n) .
$$

Dans le cas des couches minces périodiques, pour obtenir les conditions aux limites approchées nous écrivons $\left.\partial_{y} \tilde{u}_{i}^{\delta, j}(s, y)\right|_{y=0}$ en fonction de $\left.\tilde{u}_{i}^{\delta, j}(s, y)\right|_{y=0}$ et de ses dérivées par rapport à la variable tangentielle $s$.

En prenant la moyenne par rapport à $x$ dans les équations $(15,16)$ et en utilisant les conditions aux limites $v_{i}^{j}=0$ sur $y=0$ et $\partial_{y} v_{i}^{j}=0$ sur $y=-1$, on obtient

$$
\begin{gathered}
\left.\partial_{y} \tilde{u}_{i}^{0}(s, y)\right|_{y=0}=0, \\
\left.\partial_{y} \tilde{u}_{i}^{1}(s, y)\right|_{y=0}=0 .
\end{gathered}
$$

Ceci donne (en gardant les mêmes notations que dans la section précédente)

- Condition aux limites approchée d'ordre 0 :

$$
\partial_{n} \tilde{u}_{e}^{\delta, 0}=0 \quad \text { et } \partial_{n} \tilde{u}_{e}^{\delta, 1}=0 \quad \text { sur } \partial \Omega .
$$

La condition aux limites approchée d'ordre zéro pour les couches minces périodiques n'est donc pas affectée par les variations rapides de la perméabilité magnétique suivant la direction tangentielle $s$. Pour trouver la condition aux limites approchées d'ordre 1, nous prenons la moyenne dans l'équation (17) par rapport à la variable supplémentaire $x$. Ceci donne :

$$
\partial_{y}^{2} \tilde{u}_{i}^{2}(s, y)=\partial_{y}^{2} \tilde{v}_{i}^{2}(s, y)=-\left.\partial_{s}^{2} u_{e}^{0}(s, n)\right|_{n=0}-\left.k^{2}\left(\int_{-1}^{0} q(s, x, y) \mathrm{d} x\right) u_{e}^{0}(s, n)\right|_{n=0} .
$$

Maintenant, compte-tenu des identités

$$
\begin{aligned}
& \left.\tilde{u}_{i}^{0}(s, y)\right|_{y=0}=\left.u_{e}^{0}(s, n)\right|_{n=0}, \\
& \left.\tilde{u}_{i}^{1}(s, y)\right|_{y=0}=\left.u_{e}^{1}(s, n)\right|_{n=0},
\end{aligned}
$$

on obtient :

- Condition aux limites approchée d'ordre 1:

$$
\partial_{n} \tilde{u}_{e}^{\delta, 2}+\delta k^{2} \tilde{q}(s) \tilde{u}_{e}^{\delta, 2}+\delta \partial_{s}^{2} \tilde{u}_{e}^{\delta, 2}=0 \quad \text { sur } \partial \Omega,
$$

où le coefficient de perméabilité magnétique effectif est donné par l'expression suivante:

$$
\tilde{q}(s)=\int_{-1}^{0} \int_{0}^{1} q(s, x, y) \mathrm{d} x \mathrm{~d} y .
$$

Il est facile de voir que l'identité précédente donne (10) si le coefficient de perméabilité magnétique $q=$ $q(s, s / \delta, n / \delta)$ est indépendant de $s / \delta$. Dans le cas des conditions aux limites de type Dirichlet sur $\{n=-\delta\}$, on peut obtenir de la même manière que le coefficient de permittivité électrique effectif est donné par

$$
\tilde{q}=3\left[-\int_{-1}^{0}\left(\int_{-1}^{y} \int_{0}^{1} q\left(s, x, y^{\prime}\right) \mathrm{d} x\left(y^{\prime}+1\right) \mathrm{d} y^{\prime}\right) \mathrm{d} y+\int_{-1}^{0} \int_{0}^{1} q(s, x, y) \mathrm{d} x(y+1) \mathrm{d} y\right]
$$


au lieu de (13).

Comme dans la première section, nous approchons dans $\Omega_{e}$ la solution du problème de transmission (14) par la solution de l'équation de Helmholtz

$$
\left\{\begin{array}{l}
\Delta \tilde{u}_{e}^{\delta, j}+k^{2} \tilde{u}_{e}^{\delta, j}=0 \quad \text { dans } \Omega_{e} \\
(18) \text { ou }(21) \quad \text { sur } \partial \Omega \\
\tilde{u}_{e}^{\delta, j}-u^{\text {in }} \text { satisfait la condition de radiation sortante }
\end{array}\right.
$$

pour $j=0,1,2$.

Pour l'étude de l'existence et l'unicité d'une solution à ces équations nous renvoyons à [14]. Nous allons dans la section suivante estimer la différence entre l'unique solution $\tilde{u}_{e}^{\delta, j}$ de $(23)$ et $u_{e}^{\delta}$ dans une norme appropriée.

\subsection{Cas d'une condition aux limites de type Dirichlet}

Lorsque la condition aux limites sur $\{n=-\delta\} \times] 0,+\infty[$ est de type Dirichlet (i.e. en polarisation transverse magnétique), ces conditions aux limites approchées sur $\partial \Omega$ deviennent :

- Condition aux limites approchée d'ordre 0 :

$$
\tilde{u}_{e}^{\delta, 0}=0
$$

- Condition aux limites approchée d'ordre 1 :

$$
\left(1+\frac{\delta}{2} c(s)\right) \tilde{u}_{e}^{\delta, 1}+\delta \partial_{n} \tilde{u}_{e}^{\delta, 1}=0
$$

- Condition aux limites approchée d'ordre 2 :

$$
\left(1+\frac{\delta}{2} c(s)\right) \tilde{u}_{e}^{\delta, 2}+\delta \partial_{n} \tilde{u}_{e}^{\delta, 2}+\frac{\delta^{2}}{3}\left\{-\partial_{s^{2}}^{2} \tilde{u}_{e}^{\delta, 2}-\left(\frac{1}{4} c^{2}(s)-\partial_{n} c(s, 0)+\tilde{q}(s) \partial_{t}^{2}\right) \tilde{u}_{e}^{\delta, 2}\right\}=0
$$

où $\tilde{q}(s)$ est donné par (13).

Remarque 3.1. Nous remarquons que la généralisation de cette approche formelle peut se faire sans trop de difficultés au régime temporel. L'équation des ondes s'écrit :

$$
\left\{\begin{array}{l}
\left.\Delta u_{i}^{\delta}-q\left(s, \frac{s}{\delta}, \frac{n}{\delta}\right) \partial_{t^{2}}^{2} u_{i}^{\delta}=0 \quad \text { dans } \Omega_{i}^{\delta} \times\right] 0,+\infty[ \\
\left.\Delta u_{e}^{\delta}-\partial_{t^{2}}^{2} u_{e}^{\delta}=0 \quad \text { dans } \Omega_{e} \times\right] 0,+\infty[ \\
\left.\partial_{n} u_{i}^{\delta}=0 \quad \operatorname{sur}\{n=-\delta\} \times\right] 0,+\infty[ \\
\left.\left.u_{i}^{\delta}\right|_{n \rightarrow 0^{-}}=\left.u_{e}^{\delta}\right|_{n \rightarrow 0^{+}},\left.\partial_{n} u_{i}^{\delta}\right|_{n \rightarrow 0^{-}}=\left.\partial_{n} u_{e}^{\delta}\right|_{n \rightarrow 0^{+}} \quad \text { sur } \partial \Omega \times\right] 0,+\infty[
\end{array}\right.
$$

(plus des conditions initiales). On peut facilement vérifier en utilisant l'Ansatz

$$
u_{i}^{\delta}(s, n ; t)=u_{i}^{0}(s, x, y ; t)+\delta u_{i}^{1}(s, x, y ; t)+\delta^{2} u_{i}^{2}(s, x, y ; t)+\ldots,
$$


où $x=s / \delta, y=n / \delta$ et $u^{j}$ est périodique en $x=s / \delta$ de période un pour tout entier naturel $j$ que les conditions aux limites approchées pour l'équation des ondes (24) sont les transformées de Fourier en la fréquence $k$ (la variable duale) des conditions aux limites approchées pour le problème fréquentiel. En particulier, les conditions aux limites approchées $(18,21)$ en polarisation TE (transverse électrique) deviennent :

- Condition aux limites approchée d'ordre 0 :

$$
\partial_{n} \tilde{u}_{e}^{\delta, 0}=0
$$

- Condition aux limites approchée d'ordre 1:

$$
\partial_{n} \tilde{u}_{e}^{\delta, 1}+\delta \partial_{s}^{2} \tilde{u}_{e}^{\delta, 1}-\delta \tilde{q}(s) \partial_{t}^{2} \tilde{u}_{e}^{\delta, 1}=0 .
$$

\section{RÉsultats De CONVERGence POUR les COUChes MinCES PÉRIOdiQues}

Notre principal objectif dans cette section est d'estimer les quantités $u^{\delta}-\tilde{u}_{e}^{\delta, 0}$ et $u^{\delta}-\tilde{u}_{e}^{\delta, 1}$ dans une norme appropriée. Nous allons tout d'abord écrire à l'aide d'un changement d'échelles une formulation variationnelle du problème de transmission (14) dans un domaine fixe (indépendant de $\delta$ ). Soit $R$ un réel positif assez grand. Soit $(r, \theta)$ un système de coordonnées polaires associé à une origine $O \in \Omega_{i}$ où $r=|x|$ la variable radiale et $\theta$ est la variable angulaire. Nous introduisons les domaines

$$
\Omega_{e}^{R}=\Omega_{e} \cap\{|x|<R\}, \quad \Omega_{\delta}^{R}=\Omega_{e}^{R} \cup \Omega_{i}^{\delta} \cup \partial \Omega
$$

et le cercle $S_{R}=\{r=R\}$ de rayon $R$ et de centre $O$. Nous utiliserons les notations usuelles pour les espaces de Sobolev. Dans toute la suite, nous noterons

$$
u^{\delta}= \begin{cases}u_{i}^{\delta} & \text { dans } \Omega_{i}^{\delta}, \\ u_{e}^{\delta} & \text { dans } \Omega_{e} .\end{cases}
$$

\subsection{Formulation variationnelle}

Rappelons la définition de l'opérateur électromagnétique extérieur :

$$
\left\{\begin{array}{l}
T_{R}: H^{1 / 2}\left(S_{R}\right) \mapsto H^{-1 / 2}\left(S_{R}\right) \\
u_{\mid S_{R}} \rightarrow \partial_{n} u_{\mid S_{R}}
\end{array}\right.
$$

où $u$ est solution de l' équations de Helmholtz $\left(\Delta+k^{2}\right) u=0$ (plus la condition de radiation sortante) dans le domaine extérieur $\{|x|>R\}$, (pour les détails voir par exemple [20]).

Lemme 4.1. L'opérateur $T_{R}$ est continu de $H^{1 / 2}\left(S_{R}\right) \mapsto H^{-1 / 2}\left(S_{R}\right)$. Il vérifie les inégalités suivantes :

$$
\begin{aligned}
\|u\|_{\mathrm{L}^{2}\left(S_{R}\right)}^{2} & \leq-\Re e\left(T_{R} u, \bar{u}\right) \leq\|u\|_{\mathrm{H}^{1 / 2}\left(S_{R}\right)}^{2}, \\
0 & \leq \Im m\left(T_{R} u, \bar{u}\right) \leq k\|u\|_{\mathrm{L}^{2}\left(S_{R}\right)}^{2}
\end{aligned}
$$

où $($,$) est le crochet de dualité H^{-1 / 2}\left(S_{R}\right), H^{1 / 2}\left(S_{R}\right)$.

Pour toute fonction test $u^{t} \in H^{1}\left(\Omega_{\delta}^{R}\right)$, nous avons

$$
\int_{\Omega_{e}^{R}} \nabla u_{e}^{\delta} \cdot \nabla u_{e}^{t}-k^{2} \int_{\Omega_{e}^{R}} u_{e}^{\delta} u_{e}^{t}-\left(T_{R}\left(u_{e}^{\delta}\right), u_{e}^{t}\right)+\int_{\Omega_{i}^{\delta}} \nabla u_{i}^{\delta} \cdot \nabla u_{i}^{t}-k^{2} \int_{\Omega_{i}^{\delta}} q^{\delta} u_{i}^{\delta} u_{i}^{t}=\left(f^{\mathrm{in}}, u^{t}\right),
$$


où $q^{\delta}=q(s, s / \delta, n / \delta), u^{t}=u_{e}^{t}$ dans $\Omega_{e}^{R}, u^{t}=u_{i}^{t}$ dans $\Omega_{i}^{\delta}$ et $f^{\text {in }}=T_{R}\left(u^{\text {in }}\right)-\partial_{r} u_{\mid S_{R}}^{\text {in }}$. Pour toute fonction $u_{i}$ définie dans la couche mince $\Omega_{i}^{\delta}$, nous noterons $\hat{u}_{i}(y)=u_{i}(\delta y)$, pour tout $\left.y \in\right]-1,0[$. On écrit que

$$
\int_{\Omega_{i}^{\delta}} \nabla u_{i}^{\delta} \cdot \nabla u_{i}^{t}=\delta \int_{\partial \Omega} \int_{-1}^{0}(1+\delta y c(s))^{-1} \partial_{s} \hat{u}_{i}^{\delta} \partial_{s} \hat{u}_{i}^{t} \mathrm{~d} y \mathrm{~d} s+\delta^{-1} \int_{\partial \Omega} \int_{-1}^{0}(1+\delta y c(s)) \partial_{y} \hat{u}_{i}^{\delta} \partial_{y} \hat{u}_{i}^{t} \mathrm{~d} y \mathrm{~d} s
$$

et

$$
\int_{\Omega_{i}^{\delta}} q u_{i}^{\delta} u_{i}^{t}=\delta \int_{\partial \Omega} \int_{-1}^{0}(1+\delta y c(s)) q\left(s, \frac{s}{\delta}, y\right) \hat{u}_{i}^{\delta} \hat{u}_{i}^{t} \mathrm{~d} y \mathrm{~d} s .
$$

L'équation variationnelle (25) s'écrit alors

$$
\begin{aligned}
\int_{\Omega_{e}^{R}} \nabla u_{e}^{\delta} \cdot \nabla & u_{e}^{t}-k^{2} \int_{\Omega_{e}^{R}} u_{e}^{\delta} u_{e}^{t}-\left(T_{R}\left(u_{e}^{\delta}\right), u_{e}^{t}\right)+\delta \int_{\partial \Omega} \int_{-1}^{0}(1+\delta y c(s))^{-1} \partial_{s} \hat{u}_{i}^{\delta} \partial_{s} \hat{u}_{i}^{t} \mathrm{~d} y \mathrm{~d} s \\
& +\delta^{-1} \int_{\partial \Omega} \int_{-1}^{0}(1+\delta y c(s)) \partial_{y} \hat{u}_{i}^{\delta} \partial_{y} \hat{u}_{i}^{t}-\delta k^{2} \int_{\partial \Omega} \int_{-1}^{0}(1+\delta y c(s)) q\left(s, \frac{s}{\delta}, y\right) \hat{u}_{i}^{\delta} \hat{u}_{i}^{t}=\left(f^{\text {in }}, u_{e}^{t}\right) .
\end{aligned}
$$

Théorème 4.1. La formulation variationnelle (28) admet une unique solution $\left(u_{e}^{\delta}, \hat{u}_{i}^{\delta}\right) \in H^{1}\left(\Omega_{e}^{R}\right) \times H^{1}(]-$ $1,0[\times \partial \Omega)$ telle que $\left.u_{e}^{\delta}\right|_{n=0}=\left.\hat{u}_{i}^{\delta}\right|_{y=0}$ et $\left.\partial_{n} u_{e}^{\delta}\right|_{n=0}=\left.\delta \partial_{y} \hat{u}_{i}^{\delta}\right|_{y=0}$.

Preuve. Les principaux arguments, utilisés par Bendali-Lamrabet pour démontrer le résultat équivalent dans le cas d'une couche homogène, restent valables grâce au fait que la fonction $q$ soit bornée par rapport aux variables $s$ et $s / \delta$.

\subsection{Résultats de convergence et estimation d'erreur}

Soit maintenant $u_{e}^{0}$ défini comme l'unique solution dans $H^{1}\left(\Omega_{e}^{R}\right)$ de l'équation variationnelle :

$$
\forall u_{e}^{t} \in H^{1}\left(\Omega_{e}^{R}\right), \quad \int_{\Omega_{e}^{R}} \nabla u_{e}^{0} \cdot \nabla u_{e}^{t}-k^{2} \int_{\Omega_{e}^{R}} u_{e}^{0} u_{e}^{t}-\left(T_{R}\left(u_{e}^{0}\right), u_{e}^{t}\right)=\left(f^{\mathrm{in}}, u_{e}^{t}\right),
$$

et la fonction $\hat{u}_{i}^{0}$ donnée par

$$
\left.\hat{u}_{i}^{0}(s, y)=\left.u_{e}^{0}(s, n)\right|_{n=0}, \quad y \in\right]-1,0[.
$$

Remarquons que (29) correspond à la formulation variationnelle du problème (23) avec la condition (18).

Avant de montrer la convergence de $\left(u_{e}^{\delta}, \hat{u}_{i}^{\delta}\right)$ vers $\left(u_{e}^{0}, \hat{u}_{i}^{0}\right)$ lorsque $\delta$ tend vers zéro, notons que les résultats de régularité classiques de solutions de problèmes aux limites elliptiques permettent d'avoir $\left.u_{e}^{0}(s, n)\right|_{n=0} \in \mathcal{C}^{\infty}(\partial \Omega)$ si $\partial \Omega$ est de classe $\mathcal{C}^{\infty}$. Cette régularité supplémentaire (au moins une régularité $H^{2}(\partial \Omega)$ ) joue un rôle très important dans les estimations d'erreur que nous allons établir. L'hypothèse d'un bord $\partial \Omega$ régulier (au moins de classe $\mathcal{C}^{3}$ ) est donc essentielle pour mener à terme notre analyse asymptotique. Dans toute la suite, nous supposons que la fonction $q(s, x, y)$ est bornée en les variables $x, s$ et $y$. Nous avons le

Théorème 4.2. Il existe deux constantes strictement positives $\delta_{0}$ et $C$ tel que pour tout $\left.\left.\delta \in\right] 0, \delta_{0}\right]$, nous avons l'estimation d'erreur suivante :

$$
\left\|u_{e}^{\delta}-u_{e}^{0}\right\|_{H^{1}\left(\Omega_{e}^{R}\right)}+\left\|\hat{u}_{i}^{\delta}-\hat{u}_{i}^{0}\right\|_{H^{1}\left(-1,0 ; L^{2}(\partial \Omega)\right)} \leq C \delta^{1 / 2}
$$


Preuve. Remarquons tout d'abord que ce problème de convergence est un problème de perturbation singulière à cause du terme

$$
\delta \int_{\partial \Omega} \int_{-1}^{0}(1+\delta y c(s))^{-1} \partial_{s} \hat{u}_{i}^{\delta} \partial_{s} \hat{u}_{i}^{t} \mathrm{~d} y \mathrm{~d} s
$$

C'est pour cette raison que dans ce théorème nous n'avons pas une estimation de la norme de $\hat{u}_{i}^{\delta}-\hat{u}_{i}^{0}$ dans $H^{1}(]-1,0[\times \partial \Omega)$ mais seulement dans un espace plus grand.

Nous démontrons l'estimation (31) à l'aide de l'argument de stabilité de Bendali-Lemrabet. Nous rappelons le théorème $5.5 \mathrm{de}[14]$. Ce théorème nous dit que la norme dans l'espace $H^{1}\left(\Omega_{R}^{e}\right) \times H^{1}\left(-1,0 ; L^{2}(\partial \Omega)\right)$ de la solution $u^{\delta}=\left(u_{e}^{\delta}, \hat{u}_{i}^{\delta}\right)$ de la formulation variationnelle (28) avec un second membre de l'ordre de

$$
C(\delta)\left\{\delta^{1 / 2}\left(\int_{\partial \Omega} \int_{-1}^{0}\left|\partial_{s} \hat{u}_{i}^{t}\right|^{2}\right)^{1 / 2}+\delta^{-1 / 2}\left(\int_{\partial \Omega} \int_{-1}^{0}\left|\partial_{y} \hat{u}_{i}^{t}\right|^{2}\right)^{1 / 2}+\left\|u_{e}^{t}\right\|_{H^{1}\left(\Omega_{R}^{e}\right)}\right\}
$$

est de l'ordre de $C(\delta)$ où $C(\delta)$ est une constante qui dépend de $\delta$.

Nous réécrivons la formulation suivante en introduisant la différence entre (28) et (29) :

$$
\begin{gathered}
\int_{\Omega_{e}^{R}} \nabla\left(u_{e}^{\delta}-u_{e}^{0}\right) \cdot \nabla u_{e}^{t}-k^{2} \int_{\Omega_{e}^{R}}\left(u_{e}^{\delta}-u_{e}^{0}\right) u_{e}^{t}-\left(T_{R}\left(u_{e}^{\delta}-u_{e}^{0}\right), u_{e}^{t}\right)+\delta \int_{\partial \Omega} \int_{-1}^{0}(1+\delta y c(s))^{-1} \partial_{s}\left(\hat{u}_{i}^{\delta}-\hat{u}_{i}^{0}\right) \partial_{s} \hat{u}_{i}^{t} \mathrm{~d} y \mathrm{~d} s \\
+\delta^{-1} \int_{\partial \Omega} \int_{-1}^{0}(1+\delta y c(s)) \partial_{y}\left(\hat{u}_{i}^{\delta}-\hat{u}_{i}^{0}\right) \partial_{y} \hat{u}_{i}^{t}-\delta k^{2} \int_{\partial \Omega} \int_{-1}^{0}(1+\delta y c(s))\left(\int q(s, x, y) \mathrm{d} x\right)\left(\hat{u}_{i}^{\delta}-\hat{u}_{i}^{0}\right) \hat{u}_{i}^{t} \\
=\delta \int_{\partial \Omega} \int_{-1}^{0}(1+\delta y c(s))^{-1} \partial_{s} \hat{u}_{i}^{0} \partial_{s} \hat{u}_{i}^{t} \mathrm{~d} y \mathrm{~d} s \\
+\delta k^{2} \int_{\partial \Omega} \int_{-1}^{0}(1+\delta y c(s))\left[\left(\int q(s, x, y) \mathrm{d} x\right)-q\left(s, \frac{s}{\delta}, y\right]\left(\hat{u}_{i}^{\delta}-\hat{u}_{i}^{0}\right) \hat{u}_{i}^{t} . \quad(32)\right.
\end{gathered}
$$

Comme la fonction $q(s, s / \delta, y)$ est uniformément bornée en $\delta$, le second membre est inférieur ou égal à

$$
\delta^{1 / 2}\left\{\delta^{1 / 2}\left(\int_{\partial \Omega} \int_{-1}^{0}\left|\partial_{s} \hat{u}_{i}^{t}\right|^{2}\right)^{1 / 2}+\delta^{-1 / 2}\left(\int_{\partial \Omega} \int_{-1}^{0}\left|\partial_{y} \hat{u}_{i}^{t}\right|^{2}\right)^{1 / 2}+\left\|u_{e}^{t}\right\|_{H^{1}\left(\Omega_{R}^{e}\right)}\right\} .
$$

Le théorème de stabilité s'applique directement pour nous donner l'estimation (31). Comme dans [14], le terme

$$
\delta \int_{\partial \Omega} \int_{-1}^{0} \partial_{s} \hat{u}_{i}^{\delta} \partial_{s} \hat{u}_{i}^{0}
$$

est celui dont le traitement nécessite une régularité supplémentaire de la fonction $\hat{u}_{i}^{0}$ définie par (30) et donc celle de $u_{e}^{0}$ solution du problème variationnel (29).

Nous allons maintenant établir une estimation de la norme de $u^{\delta}-\tilde{u}_{e}^{\delta, 1}$ dans $H^{1}\left(-1,0 ; L^{2}(\partial \Omega)\right)$.

Nous introduisons $u_{e}^{1} \in H^{1}\left(\Omega_{e}^{R}\right)$, l'unique solution de l'équation variationnelle suivante :

$$
\begin{aligned}
\int_{\Omega_{e}^{R}} \nabla & u_{e}^{1} \cdot \nabla u_{e}^{t}-k^{2} \int_{\Omega_{e}^{R}} u_{e}^{1} u_{e}^{t}-\left(T_{R}\left(u_{e}^{1}\right), u_{e}^{t}\right)= \\
& {\left[\left.\int_{\partial \Omega} \partial_{s}^{2} u_{e}^{0}(s, n)\right|_{n=0} u_{e}^{t}+\left.k^{2} \int_{\partial \Omega}\left(\int_{-1}^{0} \int_{0}^{1} q(s, x, y) \mathrm{d} x \mathrm{~d} y\right) u_{e}^{0}(s, n)\right|_{n=0} u_{e}^{t}\right], \forall u_{e}^{t} \in H^{1}\left(\Omega_{e}^{R}\right) }
\end{aligned}
$$

et

$$
\left.\hat{u}_{i}^{1}(s, y)=\left.u_{e}^{1}(s, n)\right|_{n=0}, \quad y \in\right]-1,0[.
$$


Ce dernier système correspond à la formulation variationnelle du système $\left(u_{e}^{2}, \tilde{u}_{i}^{2}\right)$ solution de (19) obtenu lors de l'écriture formelle des conditions aux limites approchées.

Remarquons que pour définir le terme correcteur $u_{e}^{1}$ comme solution de l'équation variationnelle précédente dans $H^{1}\left(\Omega_{e}^{R}\right)$, nous utilisons la régularité $H^{2}$ (supplémentaire) de la fonction $u_{e}^{0}$ solution du problème variationnel (29).

Nous remarquons également que $u_{e}^{0}+\delta u_{e}^{1}$ est la solution de l'équation de Helmholtz (11) et qu'elle vérifie la condition aux limites approchée :

$$
\partial_{n}\left(u_{e}^{0}+\delta u_{e}^{1}\right)+\delta k^{2} \tilde{q}(s)\left(u_{e}^{0}+\delta u_{e}^{1}\right)+\delta \partial_{s}^{2}\left(u_{e}^{0}+\delta u_{e}^{1}\right)=O\left(\delta^{2}\right) \quad \text { sur } \partial \Omega
$$

Le résultat de stabilité invoqué plus loin nous permet de donner une estimation de la norme de $\tilde{u}_{e}^{\delta, 2}-\left(u_{e}^{0}+\delta u_{e}^{1}\right)$ où $\tilde{u}_{e}^{\delta, 2}$ est solution de (23) avec la condition (21).

Nous avons le

Théorème 4.3. La paire

$$
\left(\frac{u_{e}^{\delta}-\left(u_{e}^{0}+\delta u_{e}^{1}\right)}{\delta}, \frac{\hat{u}_{i}^{\delta}-\left(\hat{u}_{i}^{0}+\delta \hat{u}_{i}^{1}\right)}{\delta}\right)
$$

converge fortement vers $(0,0)$ dans $H^{1}\left(\Omega_{e}^{R}\right) \times H^{1}\left(-1,0 ; L^{2}(\partial \Omega)\right)$.

Preuve. Soit

$$
\hat{u}_{i}^{t}(s, y)=\left.\hat{u}_{i}^{t}(s, y)\right|_{y=0},
$$

une fonction test indépendante de $y$ et $u_{e}^{t} \in H^{1}\left(\Omega_{e}^{R}\right)$ telle que $\left.u_{e}^{t}\right|_{n=0}=\hat{u}_{i}^{t}$. Nous avons

$$
\left\{\begin{aligned}
\int_{\Omega_{e}^{R}} & \nabla\left(u_{e}^{\delta}-u_{e}^{0}-\delta u_{e}^{1}\right) \cdot \nabla u_{e}^{t}-k^{2} \int_{\Omega_{e}^{R}}\left(u_{e}^{\delta}-u_{e}^{0}-\delta u_{e}^{1}\right) u_{e}^{t}-\left(T_{R}\left(u_{e}^{\delta}-u_{e}^{0}-\delta u_{e}^{1}\right), u_{e}^{t}\right) \\
& +\delta \int_{\partial \Omega} \int_{-1}^{0}(1+\delta y c(s))^{-1} \partial_{s}\left(\hat{u}_{i}^{\delta}-\hat{u}_{i}^{0}-\delta \hat{u}_{i}^{1}\right) \partial_{s} \hat{u}_{i}^{t} \mathrm{~d} y \mathrm{~d} s \\
& -\delta k^{2} \int_{\partial \Omega} \int_{-1}^{0}(1+\delta y c(s)) q\left(s, \frac{s}{\delta}, y\right)\left(\hat{u}_{i}^{\delta}-\hat{u}_{i}^{0}-\delta \hat{u}_{i}^{1}\right) \hat{u}_{i}^{t} \mathrm{~d} y \mathrm{~d} s \\
= & \delta \int_{\partial \Omega} \int_{-1}^{0}(1+\delta y c(s))^{-1} \partial_{s} \hat{u}_{i}^{0} \partial_{s} \hat{u}_{i}^{t} \mathrm{~d} y \mathrm{~d} s-\left.\delta \int_{\partial \Omega} \partial_{s}^{2} u_{e}^{0}(s, n)\right|_{n=0} u_{e}^{t} \\
& -\delta k^{2} \int_{\partial \Omega} \int_{-1}^{0}(1+\delta y c(s)) q\left(s, \frac{s}{\delta}, y\right) \hat{u}_{i}^{0} \hat{u}_{i}^{t} \mathrm{~d} y \mathrm{~d} s+\left.\delta k^{2} \int_{\partial \Omega}\left(\int_{-1}^{0} \int_{0}^{1} q(s, x, y) \mathrm{d} x \mathrm{~d} y\right) u_{e}^{0}(s, n)\right|_{n=0} u_{e}^{t} \\
& +\delta^{2} \int_{\partial \Omega} \int_{-1}^{0}(1+\delta y c(s))^{-1} \partial_{s} \hat{u}_{i}^{1} \partial_{s} \hat{u}_{i}^{t} \mathrm{~d} y \mathrm{~d} s .
\end{aligned}\right.
$$

Le seul terme nouveau par rapport à la formulation de Bendali-Lemrabet pour les couches minces homogènes [14] est celui qui fait intervenir la quantité

$$
Q^{\delta}=\int_{\partial \Omega} \int_{-1}^{0} q\left(s, \frac{s}{\delta}, y\right) \hat{u}_{i}^{\delta} \hat{u}_{i}^{t} \mathrm{~d} y \mathrm{~d} s
$$


tous les autres termes se traitent d'une manière analogue. La convergence faible de $q(s, s / \delta, y)$ vers $\int_{0}^{1} q(s, x, y) \mathrm{d} x$ dans $L^{2}$ et le théorème précédent, nous permettent de démontrer que la quantité

$$
\begin{aligned}
R^{\delta}= & Q^{\delta}-\left.\int_{\partial \Omega}\left[\int_{-1}^{0} \int_{0}^{1} q(s, x, y) \mathrm{d} x \mathrm{~d} y\right] u_{e}^{0}(s, n)\right|_{n=0} \hat{u}_{i}^{t} \mathrm{~d} s \\
= & \left.\int_{\partial \Omega} \int_{-1}^{0}\left[q\left(s, \frac{s}{\delta}, y\right)-\int_{0}^{1} q(s, x, y) \mathrm{d} x \mathrm{~d} y\right] u_{e}^{0}(s, n)\right|_{n=0} \hat{u}_{i}^{t} \mathrm{~d} s \\
& +\int_{\partial \Omega} \int_{-1}^{0} q\left(s, \frac{s}{\delta}, y\right)\left[\hat{u}_{i}^{\delta}-\left.u_{e}^{0}(s, n)\right|_{n=0}\right] \hat{u}_{i}^{t} \mathrm{~d} y \mathrm{~d} s
\end{aligned}
$$

converge vers zéro. Grâce à ce résultat de convergence, l'argument de stabilité de Bendali-Lemrabet et le théorème 4.1 nous conduisent à la convergence de la paire (33) vers zéro.

Nous terminons cette démonstration par remarquer que lorsque la fonction $q$ est indépendante de la variable $x$ la situation est un peu différente. Comme la quantité

$$
q\left(s, \frac{s}{\delta}, y\right)-\int_{0}^{1} q(s, x, y) \mathrm{d} x=0
$$

on obtient que le taux de convergence vers zéro dans ce cas de la quantité (33) est de l'ordre de $\delta^{1 / 2}$ et donc, il existe deux constantes strictement positives $\delta_{0}$ et $C$ telles que pour tout $\left.\delta \in\right] 0, \delta_{0}$ ], nous avons l'estimation d'erreur suivante :

$$
\left\|u_{e}^{\delta}-\left(u_{e}^{0}+\delta u_{e}^{1}\right)\right\|_{H^{1}\left(\Omega_{e}^{R}\right)}+\left\|\hat{u}_{i}^{\delta}-\left(\hat{u}_{i}^{0}+\delta \hat{u}_{i}^{1}\right)\right\|_{H^{1}\left(-1,0 ; L^{2}(\partial \Omega)\right)} \leq C \delta^{3 / 2} .
$$

Cette estimation d'ordre 2 a été déjà obtenue par une méthode différente pour les conditions aux limites approchant une couche mince de coefficients constants contenant un réseau de petites inclusions conductrices réparties périodiquement le long de $\partial \Omega$ (voir [1]).

Remarquons que les conditions aux limites approchées (13) avec une permittivité électrique donnée par (22) dans le cas d'une condition aux limites de type Dirichlet sur $\{n=-\delta\}$ dans le problème de transmission (14) se justifient de la même manière.

Nous remarquons aussi que les estimations sont optimales pour la paire $\left(u_{e}^{\delta}, u_{i}^{\delta}\right)$, mais elles le sont moins pour $u_{e}^{\delta}$ tout seul. On obtient facilement grâce à (33) que

$$
\left\|u_{e}^{\delta}-u_{e}^{0}\right\|_{H^{1}\left(\Omega_{e}^{R}\right)} \leq C \delta
$$

\section{Conditions Aux limites ApprochéEs : CAS $\mu$ PÉRiodique}

Nous allons écrire des conditions aux limites approchées dans le cas où le coefficient de perméabilité magnétique est rapidement variable.

\subsection{Présentation du problème}

Soit $\Omega$ un ouvert borné régulier de $\mathbb{R}^{2},(s, n)$ la paramétrisation locale de son bord $\partial \Omega$ qui est supposé être de classe $\left.\mathcal{C}^{\infty}, \Omega_{i}^{\delta}=\partial \Omega \times\right]-\delta, 0\left[\right.$ et $\Omega_{e}$ le complémentaire de $\bar{\Omega}$ dans $\mathbb{R}^{2}$. Nous considérons le problème 
de transmission suivant :

$$
\left\{\begin{array}{l}
\operatorname{div}\left(\frac{1}{\mu(s / \delta, n / \delta)} \nabla u_{i}^{\delta}\right)+k^{2} u_{i}^{\delta}=0 \quad \text { in } \Omega_{i}^{\delta}, \\
\frac{1}{\mu_{0}} \Delta u_{e}^{\delta}+k^{2} u_{e}^{\delta}=0 \quad \text { in } \Omega_{e}, \\
\partial_{n} u_{i}^{\delta}=0 \quad \operatorname{sur}\{n=-\delta\}, \\
\left.u_{i}^{\delta}\right|_{n \rightarrow 0^{-}}=\left.u_{e}^{\delta}\right|_{n \rightarrow 0^{+}},\left.\frac{1}{\mu(s / \delta, n / \delta)} \partial_{n} u_{i}^{\delta}\right|_{n \rightarrow 0^{-}}=\left.\frac{1}{\mu_{0}} \partial_{n} u_{e}^{\delta}\right|_{n \rightarrow 0^{+}}, \\
u_{e}^{\delta}-u^{\text {in }} \text { satisfaisant une condition de radiation sortante, }
\end{array}\right.
$$

où la perméabilité magnétique $\mu=f(s / \delta), f$ périodique de période 1 , et $\mu=\mu_{0}$ à l'extérieur de la couche mince $\Omega_{i}^{\delta}$. Pour écrire les conditions aux limites approchées, nous utilisons une Ansatz dans la couche mince. Nous exprimons $u_{i}^{\delta}(s, n)$, la solution du problème aux limites (35), formellement en série de puissance de $\delta$ sous la forme :

$$
u_{i}^{\delta}(s, n)=u_{i}^{0}(s, x, y)_{\mid x=s / \delta, y=n / \delta}+\delta u_{i}^{1}(s, x, y)_{\mid x=s / \delta, y=n / \delta}+\ldots
$$

où $u_{i}^{j}$ est périodique par rapport à la variable rapide $x=s / \delta$ de période 1 pour tout entier $j$. Nous posons $u_{i}^{\delta, j}$ l'asymptotique tronquée à l'ordre $j$ et cherchons $u_{e}^{\delta}$ sous la forme :

$$
u_{e}^{\delta}(s, n)=u_{e}^{0}(s, n)+\delta u_{e}^{1}(s, n)+\delta^{2} u_{e}^{2}(s, n)+\ldots
$$

\subsection{Une nouvelle écriture}

Nous considérons, vu les problèmes relatifs à la condition de transmission, une nouvelle écriture du problème. Nous allons élargir la couche, faisant passer le domaine intérieur de $]-\delta, 0[$ à $]-\delta, \delta[$ ou encore après décalage à ] $-2 \delta, 0[$. Le choix de la distance $\delta$ étant arbitraire, nous allons le justifier rigoureusement plus loin. Le but de ce changement de domaine, est d'éviter d'écrire les conditions aux limites approchées en $n=-\delta$ où on a un saut de $\mu$. Notre nouveau problème de transmission s'écrit :

$$
\left\{\begin{array}{l}
\left.\operatorname{div}\left(\frac{1}{\mu(s / \delta, n / \delta)} \nabla u_{i}^{\delta}\right)+k^{2} u_{i}^{\delta}=0 \text { pour } n \in\right]-2 \delta, 0[, \\
\frac{1}{\mu_{0}} \Delta u_{e}^{\delta}+k^{2} u_{e}^{\delta}=0 \quad \text { dans } n>0, \\
u_{i}^{\delta}=0 \quad \operatorname{pour}\{n=-2 \delta\}, \\
\left.u_{i}^{\delta}\right|_{n \rightarrow 0^{-}}=\left.u_{e}^{\delta}\right|_{n \rightarrow 0^{+}}, \\
\left.\frac{1}{\mu_{0}} \partial_{n} u_{i}^{\delta}\right|_{n \rightarrow 0^{-}}=\left.\frac{1}{\mu_{0}} \partial_{n} u_{e}^{\delta}\right|_{n \rightarrow 0^{+}}, \\
u_{e}^{\delta}-u^{\text {in }} \text { satisfaisant une condition de radiation. }
\end{array}\right.
$$

On pose $\left.\Omega_{i}^{\delta}=\right]-2 \delta, 0[\times \partial \Omega$. 


\subsection{Opérateur "Neumann-Dirichlet" sur le bord}

Contrairement à la présentation de la section précédente, nous n'allons pas essayer d'écrire l'opérateur $\operatorname{div}\left(\frac{1}{\mu} \nabla\right)$ en coordonnées locales, ni essayer de résoudre les équations différentielles qui découlent de l' identification des équations en $\delta$. Nous allons procéder autrement en introduisant un nouvel opérateur $T^{\delta}$, tel que

$$
\left\{\begin{array}{l}
T^{\delta}: H^{-1 / 2}(\partial \Omega) \rightarrow H^{1 / 2}(\partial \Omega) \\
\left.\partial_{n} u_{i}^{\delta}\right|_{n \rightarrow 0^{-}} \mapsto T^{\delta}\left(\left.\partial_{n} u_{i}^{\delta}\right|_{n \rightarrow 0^{-}}\right)=\left.u_{i}^{\delta}\right|_{n \rightarrow 0^{-}}
\end{array}\right.
$$

La définition intrinsèque de $T^{\delta}$ est donnée par le problème suivant.

À toute $f \in \mathcal{C}^{\infty}(\partial \Omega)$, on associe $w$ la solution du problème aux limites suivant :

$$
\left\{\begin{array}{l}
\operatorname{div}\left(\frac{1}{\mu(s / \delta, n / \delta)} \nabla w\right)+k^{2} w=0 \quad \text { dans } \Omega_{i}^{\delta} \\
w=0 \quad \operatorname{sur}\{n=-2 \delta\} \\
\partial_{n} w=f \quad \operatorname{sur}\{n=0\}
\end{array}\right.
$$

On pose alors $T^{\delta}(f)=\left.w\right|_{n \rightarrow 0^{-}}$. L'opérateur $T^{\delta}$ est un opérateur pseudo-différentiel. On écrit une asymptotique de l'opérateur, grâce à laquelle on pourra donner une suite de conditions aux limites sur le bord $\{n=0\}$.

\subsection{Asymptotique de $T^{\delta}$}

On écrit

$$
T^{\delta}=T^{0}+\delta T^{1}+\ldots,
$$

et on identifie

$$
\begin{gathered}
T^{0}(f)=\tilde{u_{i}^{0}}(s, y)_{\mid y=0} \\
T^{1}(f)=\tilde{u_{i}^{1}}(s, y)_{\mid y=0} .
\end{gathered}
$$

En coordonnées locales, le Laplacien s'écrit :

$$
\operatorname{div}\left(\frac{1}{\mu} \nabla\right)=\frac{1}{1+c(s) n} \partial_{s}\left(\frac{1}{1+c(s) n} \frac{1}{\mu} \partial_{s}\right)+\partial_{n}\left(\frac{1}{\mu} \partial_{n}\right)+\frac{c(s, n)}{\mu} \partial_{n} .
$$

Après scaling et introduction de la variable $x$, on a l'expression :

$$
\begin{aligned}
\operatorname{div}\left(\frac{1}{\mu} \nabla\right)= & \frac{1}{\delta^{2}}\left[\frac{1}{(1+\delta c(s) y)^{2}} \partial_{x}\left(\frac{1}{\mu} \partial_{x}\right)+\partial_{y}\left(\frac{1}{\mu} \partial_{y}\right)\right] \\
& +\frac{1}{\delta}\left[\frac{1}{(1+\delta c(s) y)^{2}} \partial_{x}\left(\frac{1}{\mu} \partial_{s}\right)+\frac{1}{1+\delta c(s) y} \partial_{s}\left(\frac{1}{1+\delta c(s) y} \frac{1}{\mu} \partial_{x}\right)+\frac{c(s, \delta y)}{\mu} \partial_{y}\right] \\
& +\frac{1}{1+\delta c(s) y} \partial_{s}\left(\frac{1}{1+\delta c(s) y} \frac{1}{\mu} \partial_{s}\right) .
\end{aligned}
$$

L'identification des termes de même ordre en $\delta$ conduit à une série d'équations. 
L'identification des termes en $\delta^{-2}$ donne :

$$
\left\{\begin{array}{l}
{\left[\partial_{x}\left(\frac{1}{\mu} \partial_{x}\right)+\partial_{y}\left(\frac{1}{\mu} \partial_{y}\right)\right] u_{i}^{0}(s, x, y)=0 \quad \text { dans } \Omega_{i}^{\delta},} \\
u_{i}^{0}(s, x, y)=0 \quad \text { sur }\{y=-2\} \\
\partial_{y} u_{i}^{0}(s, x, y)=0 \quad \operatorname{sur}\{y=0\} .
\end{array}\right.
$$

L'équation (44) admet la solution triviale comme unique solution et par conséquent :

$$
T^{0}(f)=\tilde{u_{i}^{0}}(s, y)_{\mid y=0}=0
$$

L'identification des termes d'ordre $\delta^{-1}$ nous conduit à l'équation suivante :

$$
\left\{\begin{array}{l}
{\left[\partial_{x}\left(\frac{1}{\mu} \partial_{x}\right)+\partial_{y}\left(\frac{1}{\mu} \partial_{y}\right)\right] u_{i}^{1}(s, x, y)=0 \quad \text { dans } \Omega_{i}^{\delta},} \\
u_{i}^{1}(s, x, y)=0 \quad \operatorname{sur}\{y=-2\} \\
\partial_{y} u_{i}^{1}(s, x, y)=f \quad \operatorname{sur}\{y=0\} .
\end{array}\right.
$$

La solution de l'équation (45) est $u_{i}^{1}(s, x, y)=f(s) \psi(x, y)$ où $\psi$ vérifie les équations suivantes :

$$
\left\{\begin{array}{l}
\left(\partial_{x}\left(\frac{1}{\mu} \partial_{x}\right)+\partial_{y}\left(\frac{1}{\mu} \partial_{y}\right)\right) \psi=0 \\
\psi=0 \quad \operatorname{sur}\{y=-2\} \\
\partial_{y} \psi=1 \quad \operatorname{sur}\{y=0\}
\end{array}\right.
$$

D'où

$$
T^{1}(f)(s)=f(s) \tilde{\psi}(y)_{\mid y=0} \cdot
$$

Grâce à l'identification des termes d'ordre $\delta^{0}$, on obtient le problème suivant :

$$
\left\{\begin{array}{l}
{\left[\partial_{x}\left(\frac{1}{\mu} \partial_{x}\right)+\partial_{y}\left(\frac{1}{\mu} \partial_{y}\right)\right] u_{i}^{2}(s, x, y)+\left[\partial_{x}\left(\frac{1}{\mu} \partial_{s}\right)+\partial_{s}\left(\frac{1}{\mu} \partial_{x}\right)+\frac{c(s, 0)}{\mu} \partial_{y}\right] u_{i}^{1}=0 \quad \text { dans } \Omega_{i}^{\delta}} \\
u_{i}^{2}(s, x, y)=0 \quad \operatorname{sur}\{y=-2\} \\
\partial_{y} u_{i}^{2}(s, x, y)=0 \quad \operatorname{sur}\{y=0\}
\end{array}\right.
$$

La résolution de (47) nous donne :

$$
u_{i}^{2}(s, x, y)=\partial_{s} f(s) \varphi(x, y)+f(s) \theta(x, y)
$$


avec

$$
\left\{\begin{array}{l}
\left(\partial_{x}\left(\frac{1}{\mu} \partial_{x}\right)+\partial_{y}\left(\frac{1}{\mu} \partial_{y}\right)\right) \varphi=\partial_{x}\left(\frac{1}{\mu} \psi\right)+\frac{1}{\mu} \partial_{x} \psi, \\
\varphi=0 \quad \operatorname{sur}\{y=-2\}, \\
\partial_{y} \varphi=0 \quad \operatorname{sur}\{y=0\},
\end{array}\right.
$$

et

$$
\left\{\begin{array}{l}
\left(\partial_{x}\left(\frac{1}{\mu} \partial_{x}\right)+\partial_{y}\left(\frac{1}{\mu} \partial_{y}\right)\right) \theta=\frac{c(s, 0)}{\mu} \partial_{y} \psi \\
\theta=0 \quad \text { sur }\{y=-2\} \\
\partial_{y} \theta=0 \quad \operatorname{sur}\{y=0\}
\end{array}\right.
$$

On obtient donc

$$
T^{\delta}(f)(s)=\left(\partial_{s} f(s) \varphi(x, y)+f(s) \theta(x, y)\right)
$$

Formellement, on a donc

$$
T^{\delta}(f)(s)=\delta f(s) \tilde{\psi}(y)_{\mid y=0}+\delta^{2}\left(\partial_{s} f(s) \varphi(x, y)+f(s) \theta(x, y)\right)+\ldots
$$

\subsection{Justification de l'asymptotique de $T^{\delta}$}

Dans la suite, nous justifions l'écriture de l'asymptotique de l'opérateur $T^{\delta}$. Pour cela, il suffit dans un premier temps de justifier la convergence de $\left\|T^{\delta}-T^{0}-\delta T^{1}\right\|_{H^{1 / 2}(\partial \Omega)}$ vers zéro.

À cette fin, nous commençons par démontrer que

$$
\frac{\left.u_{i}^{\delta}\right|_{y \rightarrow 0^{-}}}{\delta} \rightarrow \tilde{u_{i}^{1}}(x, y)_{\mid y=0}
$$

dans $H^{1 / 2}(\partial \Omega)$. On procède en plusieurs étapes. La première étape consiste à démontrer que $\left[u_{i}^{\delta}(s, n / \delta)\right] / \delta$ est bornée dans $H^{1 / 2}(\partial \Omega)$. On en déduit une convergence faible de $\left[u_{i}^{\delta}(s, n / \delta)\right] / \delta$ vers $w$. La dernière étape est de démontrer que $w=\tilde{u_{i}^{1}}(x, y)_{\mid y=0}$.

Posons

$$
v^{\delta}(s, y)=u_{i}^{\delta}(s, n / \delta)
$$

Nous montrons tout d'abord que la quantité $w^{\delta}=\left[v^{\delta}(s, y)_{\mid y=0}\right] / \delta$ est bornée dans $H^{1}(]-2,0\left[, L^{2}(\partial \Omega)\right)$.

Nous avons le résultat suivant :

Lemme 5.1. La quantité

$$
\gamma^{\delta}=\left\|w^{\delta}(s, y)\right\|_{H^{1}(]-2,0\left[, L^{2}(\partial \Omega)\right)}+\delta\left(\int_{\partial \Omega} \int_{-2}^{0}\left|\partial_{s} w^{\delta}\right|^{2}\right)^{1 / 2} \leq C
$$

où la constante $C$ est indépendante de $\delta$. 
Preuve. Nous allons démontrer le résultat par l'absurde. Supposons que la quantité $\gamma^{\delta}$ ne soit pas bornée, à une extraction de sous-suite près, nous pouvons supposer qu'elle converge vers l'infini.

Soit

$$
z^{\delta}=\frac{w^{\delta}}{\gamma^{\delta}} .
$$

Rappelons que $v^{\delta}(s, y)$ vérifie le problème suivant :

$$
\left\{\begin{array}{l}
\left.\frac{1}{\delta^{2}}\left[\partial_{y}\left(\frac{1}{\mu} \partial_{y}\right)\right]+\frac{1}{\delta} \frac{c(s, \delta y)}{\mu} \partial_{y}+\frac{1}{1+\delta c(s) y} \partial_{s}\left(\frac{1}{1+\delta c(s) y} \frac{1}{\mu} \partial_{s}\right) v^{\delta}+k^{2} v^{\delta}=0 \text { pour } y \in\right]-2,0[ \\
v^{\delta}=0 \operatorname{sur}\{y=-2\} \\
\frac{1}{\delta} \partial_{y} v^{\delta}=f \operatorname{sur}\{y=0\}
\end{array}\right.
$$

On multiplie l'équation vérifiée par $v^{\delta}$ par une fonction test $u^{t}$ régulière, et on intègre par parties. Sachant que l'élément de surface $\mathrm{d} \Omega_{i}^{\delta}=\delta(1+\delta c(s) y) \mathrm{d} y \mathrm{~d} s$, nous obtenons :

$$
\begin{aligned}
\frac{1}{\delta} \int(1+\delta c(s) y) \frac{1}{\mu} \partial_{y} v^{\delta}(s, y) \partial_{y} u^{t} & +\delta \int \frac{1}{\mu} \frac{1}{1+\delta c(s) y} \partial_{s} v^{\delta} \partial_{s} u^{t} \\
& -\delta \int k^{2}(1+\delta c(s) y) v^{\delta} u^{t}-\left[\frac{1}{\delta} \int_{\partial \Omega} \frac{1}{\mu} \partial_{y} v^{\delta}(s, y) u^{t}(s, y)(1+\delta c(s) y)\right]_{y=-2}^{y=0}=0
\end{aligned}
$$

Soit encore, en utilisant les conditions aux limites vérifiées par $v^{\delta}(s, y)$ :

$\frac{1}{\delta} \int(1+\delta c(s) y) \frac{1}{\mu} \partial_{y} v^{\delta}(s, y) \partial_{y} u^{t}+\delta \int \frac{1}{\mu} \frac{1}{1+\delta c(s) y} \partial_{s} v^{\delta} \partial_{s} u^{t}$

$$
-\delta \int k^{2}(1+\delta c(s) y) v^{\delta} u^{t}-\int_{\partial \Omega} \frac{1}{\mu} f u^{t}(s, y)_{\mid y=0}=0 .
$$

Nous déduisons que $w^{\delta}$ vérifie l'équation suivante :

$$
\begin{aligned}
\int(1+\delta c(s) y) \frac{1}{\mu} \partial_{y} w^{\delta}(s, y) \partial_{y} u^{t}+\delta^{2} \int \frac{1}{\mu} \frac{1}{1+\delta c(s) y} \partial_{s} w^{\delta} \partial_{s} u^{t} & \\
& -\delta^{2} \int k^{2}(1+\delta c(s) y) w^{\delta} u^{t}-\int_{\partial \Omega} \frac{1}{\mu} f u^{t}(s, y)_{\mid y=0}=0
\end{aligned}
$$

et $z^{\delta}$ vérifie l'équation :

$$
\begin{aligned}
\int(1+\delta c(s) y) \frac{1}{\mu} \partial_{y} z^{\delta}(s, y) \partial_{y} u^{t}+\delta^{2} \int \frac{1}{\mu} \frac{1}{1+\delta c(s) y} & \partial_{s} z^{\delta} \partial_{s} u^{t} \\
& =\delta^{2} \int k^{2}(1+\delta c(s) y) z^{\delta} u^{t}+\frac{1}{\gamma \delta} \int_{\partial \Omega} \frac{1}{\mu} f u^{t}(s, y)_{\mid y=0}
\end{aligned}
$$


Sachant que $\delta \partial_{s} z^{\delta}$ est bornée $L^{2}$ et de même pour $\left\|z^{\delta}(s, y)\right\|_{H^{1}(]-2,0\left[, L^{2}(\partial \Omega)\right)}$, on a la convergence faible dans $H^{1}(]-2,0\left[, L^{2}(\partial \Omega)\right)$ de $z^{\delta}$ vers $z$. Par passage à la limite dans l'équation (51), nous obtenons que $z$ vérifie :

$$
\begin{array}{rll}
{\left[\partial_{y}\left(\frac{1}{\mu} \partial_{y}\right)\right] z=0,} & \\
z=0 & \operatorname{pour}\{y=-2\}, \\
\partial_{y} z=0 & \text { pour }\{y=0\} .
\end{array}
$$

D'où $z=0$. On a donc que

$$
\left\|z^{\delta}(s, y)\right\|_{L^{2}(]-2,0\left[, L^{2}(\partial \Omega)\right)} \rightarrow 0 .
$$

Il nous reste à prouver que la convergence est forte et non pas uniquement faible.

En choisissant $u^{t}=\overline{z^{\delta}}$ dans la formulation variationnelle (51), nous obtenons

$$
\begin{aligned}
\int_{\partial \Omega} \int_{-2}^{0} \frac{1}{(1+\delta c(s) y)} \frac{1}{\mu}\left|\partial_{y} z^{\delta}(s, y)\right|^{2} & \rightarrow 0 \\
\delta^{2} \int_{\partial \Omega} \int_{-2}^{0}(1+\delta c(s) y) \frac{1}{\mu}\left|\partial_{s} z^{\delta}\right|^{2} & \rightarrow 0 .
\end{aligned}
$$

Ce qui constitue une contradiction avec le fait que

$$
\left\|z^{\delta}(s, y)\right\|_{H^{1}(]-2,0\left[, L^{2}(\partial \Omega)\right)}+\delta\left(\int_{\partial \Omega} \int_{-2}^{0}\left|\partial_{s} z^{\delta}\right|^{2}\right)^{1 / 2}=1
$$

Nous sommes en mesure d'énoncer le résultat suivant :

Théorème 5.1. $\left[v^{\delta}(s, y)_{\mid y=0}\right] / \delta$ converge vers $\tilde{u}_{i}^{1}(s, y)_{\mid y=0}$ dans $H^{1 / 2}(] 0,1[)$ faible.

Preuve. On a démontré que $\left[v^{\delta}(s, y)\right] / \delta$ est bornée dans $H^{1}(]-2,0\left[, L^{2}(\partial \Omega)\right)$, donc elle converge $H^{1}(]-$ $2,0\left[, L^{2}(\partial \Omega)\right)$ faible vers un certain $w$ dans $H^{1}(]-2,0\left[, L^{2}(\partial \Omega)\right)$.

On écrit l'équation variationnelle vérifiée par $u_{i}^{\delta} / \delta$ et on choisit $\psi(s / \delta, y) u^{t}$ comme fonction test. Après deux intégrations par parties, un choix d'une fonction $u^{t}$ indépendante de $y$ et de classe $C^{\infty}([0,1] \times[-2,0])$, on obtient que les termes de bords sur $y=0$ vérifient :

$$
\left(\frac{u_{i}^{\delta}}{\delta}, \frac{1}{\mu_{0}} \partial_{y} \psi \cdot u^{t}\right)_{\mid y=0}+\left(\frac{1}{\mu_{0}} \partial_{n} u_{i}^{\delta}, \psi \cdot u^{t}\right)_{\mid y=0} \rightarrow 0 .
$$

Or, $\partial_{n} u_{i}^{\delta}=f$ et $\partial_{y} \psi=1$ sur $n=y=0$.

Soit par passage à la limite :

$$
w_{\mid y=0}=f \int \psi_{\mid y=0}=\tilde{u_{i}^{1}}(x, y)_{\mid y=0} .
$$




\subsection{Problèmes aux limites et conditions aux limites approchées}

Nous allons donner ici les problèmes aux limites associés aux conditions aux limites approchées qu'on vient d'écrire formellement. On a

$$
\left.u_{i}^{\delta}\right|_{n \rightarrow 0^{-}}=T^{\delta}\left(\left.\partial_{n} u_{i}^{\delta}\right|_{n \rightarrow 0^{-}}\right) .
$$

En remplaçant $T^{\delta}$ par son asymptotique et $u_{i}^{\delta}$ par la sienne, on obtient les relations suivantes :

$$
\tilde{u_{i}^{0}}(s, y)_{\mid y=0}=\tilde{\psi}(y)_{\mid y=0} \partial_{y} \tilde{u_{i}^{0}}(s, y)_{\mid y=0} .
$$

On obtient alors la première condition aux limites approchée :

$$
\tilde{u}_{i}^{\delta}(s, y)_{\mid y=0}=\delta \tilde{\psi}(y)_{\mid y=0} \partial_{n} \tilde{u_{i}^{\delta}}(s, y)_{\mid y=0} .
$$

En identifiant les termes d'ordre $\delta$, on obtient :

$$
T^{2}\left(\partial_{y} \tilde{u_{i}^{0}}(s, y)_{\mid y=0}\right)+T^{1}\left(\partial_{y} \tilde{u_{1}^{1}}(s, y)_{\mid y=0}\right)=\tilde{u_{1}^{1}}(s, y)_{\mid y=0} .
$$

On écrit alors la condition aux limites approchée du second ordre :

$$
\tilde{u}_{i}^{\delta}(s, y)_{\mid y=0}=\delta \tilde{\psi}(y)_{\mid y=0} \partial_{n} \tilde{u_{i}^{\delta}}(s, y)_{\mid y=0}
$$

qui est exactement la même que la condition du premier ordre.

La condition d'ordre 3 nécessite l'écriture de $T^{3}$ mais comme $u_{i}^{0}(s, y)=0$, on a la condition suivante :

$$
T^{2}\left(\partial_{y} \tilde{u}_{i}^{1}(s, y)_{\mid y=0}\right)+T^{1}\left(\partial_{y} \tilde{u}_{i}^{2}(s, y)_{\mid y=0}\right)=\tilde{u_{i}^{2}}(s, y)_{\mid y=0} .
$$

La condition du troisième ordre s'écrit :

$$
\tilde{u_{i}^{\delta}}(s, y)_{\mid y=0}=\delta \tilde{\psi}(y)_{\mid y=0} \partial_{n} \tilde{u_{i}^{\delta}}(s, y)_{\mid y=0}+\delta^{2} \tilde{\theta}(x, y) \partial_{n} \tilde{u_{i}^{\delta}}(s, y)_{\mid y=0}+\delta^{2} \tilde{\varphi}(x, y) \partial_{s} \partial_{n} \tilde{u_{i}^{\delta}}(s, y)_{\mid y=0} .
$$

Remarquons que dans le cas où $\mu$ est constante on retrouve exactement les conditions d'Enquist-Nédélec [16].

\section{BIBLIOGRAPHIE}

[1] T. Abboud et H. Ammari, Diffraction at a curved grating: TM and TE cases, Homogenization. J. Math. Anal. Appl. 202 (1996) 995-1026.

[2] T. Abboud et H. Ammari, Diffraction at a curved grating: Approximation by an infinite plane grating. J. Math. Anal. Appl. 202 (1996) 1076-1100.

[3] T. Abboud et H. Ammari, Diffraction par un réseau courbe bipériodique: Homogénéisation. C.R. Acad. Sci. Paris Sér. I 320 (1995) 301-306.

[4] T. Abboud, H. Ammari et G. Lebeau, Analyse asymptotique de la diffraction par un réseau courbe. C.R. Acad. Sci. Paris Sér. I 320 (1995) 1085-1090.

[5] H. Ammari, Scattering of waves by thin periodic layers at high frequencies using the on-surface radiation condition method. IMA J. Appl. Math. 60 (1998) 199-214.

[6] H. Ammari et S. He, Generalized effective impedance boundary conditions for an inhomogeneous thin layer in electromagnetic scattering. J. Electromagn. Waves Appl. 11 (1997) 1197-1212.

[7] H. Ammari et S. He, Homogenization and scattering for gratings. J. Electromagn. Waves Appl. 11 (1997) 1669-1683.

[8] H. Ammari et S. He, Effective impedance boundary conditions for an inhomogeneous thin layer on a curved metallic surface. IEEE Trans. Antennas Propag. 46 (1998) 710-715.

[9] H. Ammari et J.-C. Nédélec, Sur les conditions d'impédance généralisées pour les couches minces. C.R. Acad. Sci. Paris Sér. I 322 (1996) 995-1000. 
[10] H. Ammari et J.-C. Nédélec, Time-harmonic electromagnetic fields in thin chiral layers. SIAM J. Math. Anal. 29 (1998) 395-423.

[11] H. Ammari et J.-C. Nédélec, Generalized impedance boundary conditions for the Maxwell equations as singular perturbation problems. Comm. Partial Differential Equations 24 (1999) 821-850.

[12] J.F. Ahner et H.W. Wiener, On an exterior Laplace equation problem with Robin boundary condition. J. Math. Anal. Appl. 157 (1991) 127-146.

[13] A. Bendali et L. Vernhet, Résolution par éléments finis de frontière d'un problème de diffraction d'onde comportant une condition aux limites d'impédance généralisées. C.R. Acad. Sci. Paris Sér. I 321 (1995) 791-797.

[14] A. Bendali et K. Lemrabet, The effect of a thin coating on the scattering of a time-harmonic wave for the Helmholtz equation. SIAM J. Appl. Math. 56 (1996) 1664-1693.

[15] A. Bensoussan, J.L. Lions et G. Papanicolaou, Asymptotic Analysis for Periodic Structures. North Holland (1978).

[16] B. Engquist et J.-C. Nédélec, Effective boundary conditions for acoustic and electromagnetic scattering thin layers. Rapport Interne du Centre de Mathématiques Appliquées de l'École Polytechnique, 278 (1993).

[17] R.F. Harrington et J.R. Mautz, An impedance sheet approximation for thin dielectric shells. IEEE Trans. Antennas Propag. 23 (1975) 531-534.

[18] S.N. Karp et F.C. Karal, Generalized impedance boundary conditions for surface wave structures, in Electromagnetic Wave Theory, Part I, J. Brown Ed., Pergamon Press, New York (1965) 479-483.

[19] R.J. King et J.R. Wait, Electromagnetic groundwave propagation theory and experiment, in Symposia Mathematica, Vol. 17, Academic Press, New-York (1976) 107-208.

[20] J.-C. Nédélec, Ondes acoustiques et électromagnétiques, Équations intégrales, Cours de DEA, École Polytechnique (1996).

[21] T.B.A. Senior et J.L. Volakis, Approximate boundary conditions in electromagnetics. IEE Electromagnetic Waves Series 41, IEE Publication, London (1995). 